\title{
UM MODELO PARA A SUPERFÍCIE LÍQUIDA NO ESTUDO DA DINÂMICA DO ESPALHAMENTO DE Xe E Ne PELO ESQUALANO
}

\author{
Alexandre S. Leal ${ }^{* \#}$, Ivanildo Rodrigues e Heloiza H. R. Schor \\ Departamento de Química, Instituto de Ciências Exatas, Universidade Federal de Minas Gerais, \\ CP 702, 31270-901 Belo Horizonte - MG
}

Recebido em 28/3/03; aceito em 10/9/03

\begin{abstract}
A MODEL FOR THE LIQUID SURFACE IN THE STUDY OF THE DYNAMICS OF THE SCATTERING OF Xe AND Ne FROM SQUALANE. In this work we present a theoretical model to investigate the scattering of $\mathrm{Xe}$ and $\mathrm{Ne}$ by a liquid squalane surface. The liquid surface is modeled as a grid of harmonic oscillators with frequencies adjusted to experimental vibration as frequencies of the liquid squalane and the atom-surface interaction potential is modeled by a Lennard-Jones function. The three dimensional description of the dynamics of the process which occurs at the gas-liquid interface is obtained by the classical trajectory method. The general characteristics of the dynamics of the scattering process are in good agreement with experimental data.
\end{abstract}

Keywords: gas-liquid scattering; liquid surface; classical trajectories.

\section{INTRODUÇÃO}

Nos últimos anos, o estudo da interface gás-líquido tem recebido atenção crescente de pesquisadores teóricos e experimentais motivados pelo enorme potencial de aplicação tecnológica desse sistema em diversas áreas do conhecimento ${ }^{1}$. Um dos exemplos mais relevantes são os fenômenos que ocorrem na atmosfera, onde a determinação acurada de parâmetros críticos é essencial para o desenvolvimento de modelos teóricos com capacidade preditiva. Um importante processo que ocorre nesse meio é a captura não reativa de moléculas gasosas por aerosóis e gotas de água nas nuvens ${ }^{2}$. Esse processo consiste na difusão do gás para a interface líquido/gás, o transporte através desta e a posterior difusão na fase aquosa. Um exemplo típico, estudado por Jayne e colaboradores ${ }^{3}$ é a captura do formaldeído gasoso pela superfície de gotículas dos ácidos nítrico e sulfúrico em solução aquosa. Esse sistema tem um papel fundamental na fotoquímica da atmosfera, pois o $\mathrm{CH}_{2} \mathrm{O}$ pode ser removido da fase gasosa nas nuvens resultando em um aumento significativo de sua concentração na chuva, neve e neblina. Além disso, a reação do $\mathrm{CH}_{2} \mathrm{O}$ com radicais hidroxila produz ácido fórmico $(\mathrm{HCOOH})$ e contribui para a acidez da água de chuva. Outro exemplo típico é a influência de uma variedade de gases na concentração de ozônio na atmosfera, investigada por Nathanson e colaboradores ${ }^{4}$. Segundo os autores, espécies como $\mathrm{OH}, \mathrm{NO}, \mathrm{Cl}$ e $\mathrm{Br}$ podem destruir a camada de ozônio através de processos catalíticos com velocidades que, freqüentemente, excedem as de sua produção e podem ser interrompidos quando os catalisadores são convertidos em espécies relativamente inertes como $\mathrm{HCl}$ e $\mathrm{ClONO}_{2}$. Entretanto, estas espécies mais estáveis podem se tornar também reativas através de reações com aerosóis na estratosfera, como o $\mathrm{H}_{2} \mathrm{SO}_{4}$. Estudos realizados por Heal e colaboradores ${ }^{5}$ evidenciam a importância do conhecimento do coeficiente de acomodação, parâmetro que reflete a taxa de transferência de massa do gás para a fase líquida, para a avaliação das possibilidades de remoção de hidrocarbonetos da troposfera. A determinação desse parâmetro

*e-mail: asleal@cdtn.br

\# Endereço atual: Centro de Desenvolvimento da Tecnologia Nuclear, R. Mário Werneck, s/n, 30123-970 Belo Horizonte - MG é também crucial para melhor compreensão da química da superfície dos oceanos, onde o fluxo de gases como $\mathrm{SO}_{2}, \mathrm{~N}_{2} \mathrm{O}, \mathrm{CO}, \mathrm{CH}_{4}$ e outros, através da interface gás-líquido pode ser estimado teoricamente através de cálculos de hidrodinâmica ${ }^{6}$.

A crescente disponibilidade de dados experimentais da diluição de gases em soluções aquosas tem sido importante na validação de cálculos teóricos e simulações computacionais relacionadas a diversas aplicações práticas. Estudos termodinâmicos de soluções, como gases inertes em alcanos simples, têm sido empregados em modelos preditivos para sistemas complexos, como as interações entre a água e soluto presentes em macromoléculas no meio biológico ${ }^{7}$.

O espalhamento de feixes de átomos e moléculas por superfícies líquidas, como mostra a Figura 1, é uma das técnicas empregadas na investigação da dinâmica nessa interface. Esses experimentos são limitados aos líquidos de baixa pressão de vapor, para garantir que a espécie gasosa incidente atinja a superfície do líquido e não o vapor acima dele. Como mostra a Figura, o gás atinge a superfície do líquido e após a colisão, a massa, a velocidade e o tempo de vôo no percurso até o espectrômetro são registrados para as espécies que

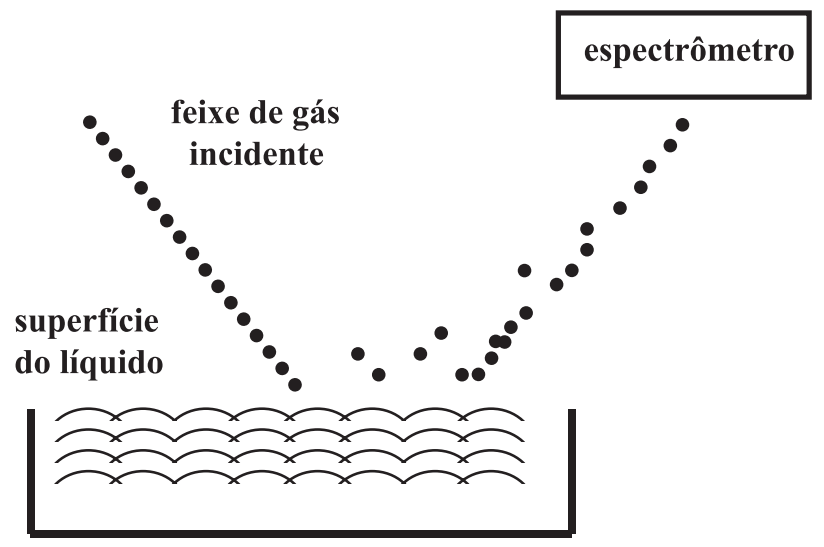

Figura 1. O gás atinge a superfície do líquido e parte das espécies espalhadas são detectadas no espectrômetro 
escapam da superfície. As espécies que atingem o espectrômetro em tempos menores têm velocidades mais altas e, portanto, transferem pouca energia para o líquido e as espécies que levam mais tempo transferem parte significativa de sua energia inicial para o líquido. Nesse caso, o gás pode ser capturado por um tempo suficientemente longo para se dissolver no líquido. Para gases não reativos, esta solvatação transiente pode ser da ordem de nanossegundos ou menos. A técnica do espalhamento, entretanto, está limitada aos líquidos de baixa pressão de vapor para garantir que a espécie gasosa incidente atinja a superfície do líquido e não o vapor acima dele.

Nas simulações realizadas por Lipkin e colaboradores ${ }^{8}$ do espalhamento de átomos de Xe pelo esqualano, a superfície do líquido é modelada como um conjunto de esferas que representam a unidade do hidrocarboneto na cadeia polimérica e os modos internos de vibração não são considerados. De modo geral, esse modelo fornece resultados satisfatórios comparados aos dados experimentais para a transferência de energia e a distribuição angular do espalhamento. Mas o percentual de átomos adsorvidos é menor que o real, resultado que, segundo os autores, se deve às restrições do modelo aos modos de absorção de energia da superfície. Os cálculos de dinâmica molecular do espalhamento de átomos de argônio sobre a superfície do In líquido, conduzidas por Tribe e colaboradores ${ }^{9}$, revelam como os diferentes tipos de colisão, a adsorção e a colisão inelástica entre o átomo e a superfície podem ser visualizados, analisando-se trajetórias típicas para cada caso. A superfície do líquido é simulada como uma caixa cúbica com um total de 864 partículas, cujos parâmetros são ajustados para fornecer os valores experimentais correspondentes da densidade e pressão de vapor. Esse modelo leva a resultados qualitativamente satisfatórios, mas o valor da tensão superficial calculada é três vezes maior que o experimental. Experiências de espalhamento de gases inertes como Xe e Ne por superfícies de líquidos poliatômicos, como esqualano e poliéterperfluorado, conduzidas por Saecker e colaboradores ${ }^{10}$ mostram que a transferência de energia na colisão depende da composição da superfície e da energia da espécie incidente. A probabilidade do gás ser adsorvido está relacionada à capacidade da superfície em absorver energia da colisão que, por sua vez, depende de seus parâmetros de constituição, como massa e modos vibracionais dos grupos protuberantes. Usando feixes de gases $\mathrm{Ne}$, Ar e Xe, Ronk e colaboradores ${ }^{11}$ investigaram a estrutura da superfície dos líquidos In, $\mathrm{Ga}$ e Bi. Um quadro comparativo dos resultados da colisão com os líquidos atômicos metálicos citados e outros líquidos poliatômicos é apresentado pelos autores, que também mostram como a transferência de energia e a intensidade do espalhamento monitorada em um ângulo fixo dependem da identidade das espécies que colidem, da energia do gás incidente e da temperatura do líquido. Em particular, a dependência na temperatura pode ser analisada considerando os deslocamentos dos átomos da superfície metálica, usando um modelo de osciladores harmônicos.

Quando um gás molecular ou atômico colide com uma superfície líquida pode ocorrer o espalhamento inelástico, onde uma pequena fração da energia do gás é trocada com a superfície, ou há a captura e dessorção posterior do gás na superfície, em um processo de múltiplas colisões. No caso do gás molecular, as moléculas podem ainda trocar sua energia interna com a superfície. A variação temporal da distância entre o gás e a superfície nos dois casos, para o tratamento do espalhamento em duas dimensões, está ilustrada na Figura 2.

Após a colisão, a espécie que colide pode não ter energia cinética suficiente para escapar, podendo ser capturada pela superfície e atravessá-la em direção ao volume do líquido, no processo de dissolução, ou ainda reagir com outras espécies adsorvidas. Ocorrem reações também na superfície, localizadas na região do impacto. Depois de algum tempo, as espécies adsorvidas, incluindo os produtos

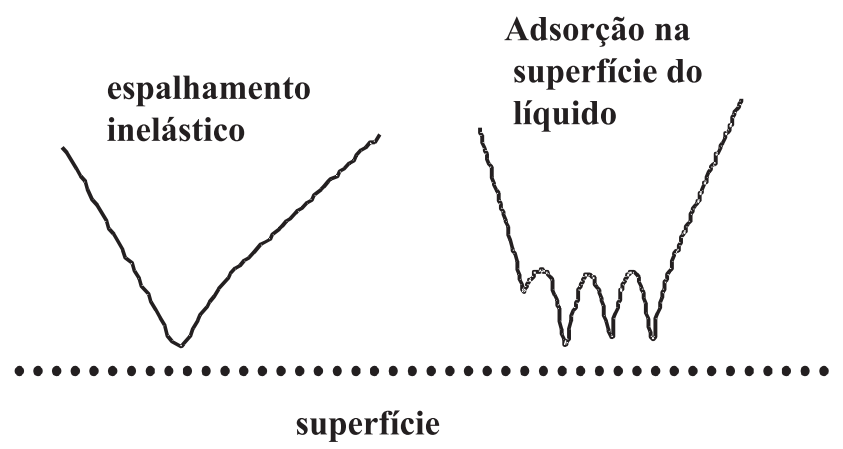

Figura 2. Trajetória de moléculas de iodo espalhadas pela superfície do líquido esqualano. À esquerda: a energia inicial da molécula é de $40 \mathrm{~kJ} /$ mol e a colisão é predominantemente inelástica. À direita: a energia inicial da molécula, que sofre várias colisões na superfície, é de $10 \mathrm{~kJ} / \mathrm{mol}$ o que caracteriza o processo de captura e posterior dessorção do gás na superfície, representada pela linha horizontal

de reação, podem adquirir energia e voltar à fase gasosa. Outras espécies podem ser ejetadas diretamente para a fase gasosa pela energia liberada na reação. Essas possibilidades de interação sugerem que os processos de transferência de energia, difusão e reação estão intrinsecamente relacionados. A investigação da dinâmica da interação gás-líquido busca compreender como esses processos ocorrem e se relacionam.

Neste trabalho, é proposto um modelo simples de representação da superfície do líquido polimérico esqualano, $\left(\mathrm{C}_{30} \mathrm{H}_{62}\right)$, como uma malha de osciladores harmônicos, que permite descrever as principais caraterísticas dinâmicas do espalhamento de átomos de Xe e Ne pela superfície.

\section{A DINÂMICA DO ESPALHAMENTO}

\section{Modelo de superfície}

O modelo proposto para a superfície do líquido considera que, do ponto de vista da dinâmica do espalhamento, ela pode ser representada pelos movimento de seus grupos proeminentes que, no caso do esqualano, cuja estrutura está representada na Figura 3a, são grupos $\mathrm{CH}_{3}$. Esta suposição é sugerida pelos resultados experimentais obtidos por Keynon e colaboradores ${ }^{12,13}$ do espalhamento de moléculas de $\mathrm{I}_{2}$ sobre a superfície de líquidos poliméricos. Nesse experimento, a molécula é usada como uma sonda para investigar as características de cada superfície, através dos seus espectros, vibracional e rotacional, de fluorescência induzida por laser. As caraterísticas dinâmicas gerais observadas neste experimento foram satisfatoriamente reproduzidas por Leal e colaboradores ${ }^{14,15} \mathrm{em}$ um modelo onde o potencial de interação molécula-superfície inclui as interações de três corpos e para o líquido é empregada uma superfície bidimensional. O modelo aqui proposto para o espalhamento átomo-superfície em três dimensões é mais simples e inclui mais graus de liberdade para a descrição da superfície líquida.

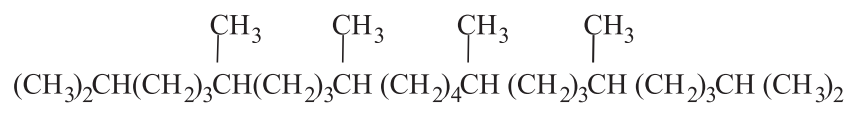

Figura 3a. Estrutura da molécula do esqualano

A superfície do esqualano líquido é modelada como um conjunto de osciladores harmônicos e quatro grupos de massa $\mathrm{M}$ equivalentes à massa do grupo $\mathrm{CH}_{3}$, como mostra a Figura 3b. As distânci- 


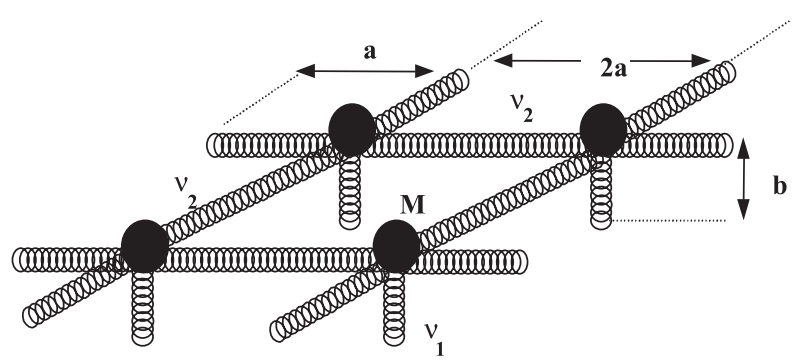

Figura 3b. Modelo esquemático para a superfície do esqualano. As esferas de massa $\mathrm{M}$ correspondem aos grupos $-\mathrm{CH}_{3}$ da Figura $3 a ; v_{1}$ e $v_{2}$ são as freqüências de oscilação e $2 a$ é a distância entre grupos adjacentes

as de equilíbrio a e b representam a separação média entre os grupos $\mathrm{CH}_{3}$ adjacentes e foram calculadas através dos valores dos comprimentos das ligações entre os átomos componentes dos grupos proeminentes na cadeia do polímero. Os valores das freqüências, $v_{1}=$ $1000 \mathrm{~cm}^{-1} \mathrm{e} \mathrm{v}_{2}=820 \mathrm{~cm}^{-1}$, de movimento dos osciladores foram inicialmente atribuídas, respectivamente, aos valores das frequiências de estiramento da ligação $\mathrm{C}-\mathrm{CH}_{3}$ e "rocking" do grupo $\mathrm{CH}_{3}$ obtidos da literatura ${ }^{16}$ e pelo espectro Raman do líquido, mostrado na Figura 4. $\mathrm{O}$ pico na região de $800 \mathrm{~cm}^{-1}$ pode ser atribuído a $v_{2}$ e o pico na região de aproximadamente $1000 \mathrm{~cm}^{-1} \mathrm{a}_{1}$.

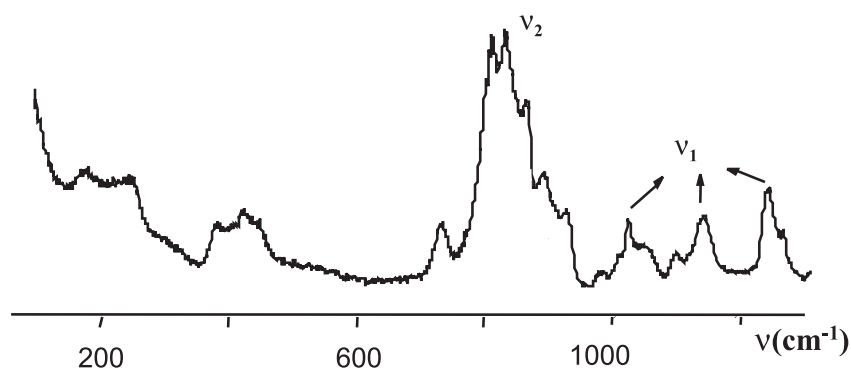

Figura 4. Espectro Raman do esqualano na região das freqüencias (em $\mathrm{cm}^{-1}$ ) de oscilação $\left(v_{1} \cong 1000\right)$ e $\left(v_{2} \cong 820\right)$ do grupo $-\mathrm{CH}_{3}$

Esse modelo representativo da superfície pode ser facilmente ampliado, acrescentando-se mais corpos e osciladores e pode ser útil na descrição do espalhamento de gás por outros tipos de superfície, como a de líquidos metálicos e sólidos ordenados, para os quais há um expressivo número de resultados teóricos e experimentais disponíveis ${ }^{17}$.

\section{O potencial de interação}

O potencial de interação átomo-superfície empregado é do tipo Lennard-Jones (LJ), representado na Equação 1,

$V_{I N T}=\sum_{i=1}^{4} 4 \varepsilon\left[\left(\frac{\sigma}{r_{i}}\right)^{12}-\left(\frac{\sigma}{r_{i}}\right)^{6}\right]$

onde $r_{i}$ é a distância do átomo à cada uma das partículas da superfície, os parâmetros $\varepsilon$ e $\sigma$ correspondem, respectivamente, à profundidade do mínimo do potencial e à distância de equilíbrio. Para estes parâmetros foram empregados valores obtidos diretamente da literatura para os sistemas gasosos $\mathrm{Xe}-\mathrm{CH}_{4} \mathrm{e} \mathrm{Ne}-\mathrm{CH}_{4}$ com os seguintes valores, respectivamente, $\left(\varepsilon(\mathrm{ev})=19,6 \times 10^{-3}, \sigma(\AA)=3,77\right),(\varepsilon(\mathrm{ev})=$ $\left.5,25 \times 10^{-3}, \sigma(\AA)=3,37\right)^{18}$.

O potencial de interação do tipo LJ é utilizado por Nathanson e colaboradores $^{8,9,19}$ na interpretação de resultados experimentais do espalhamento de átomos por superfícies líquidas para sistemas não reativos e esta escolha facilita a comparação com resultados existentes na literatura. É importante mencionar, entretanto, que o problema da determinação de potenciais mais realísticos para a interação gás-líquido fora do equilíbrio não é, de forma alguma, trivial. Em parte, por ser esta ainda uma área de estudo relativamente recente com um volume de dados experimentais ainda pequeno. Diferentemente da interação gás-sólido, onde quase três décadas de estudos fornecem uma quantidade de informação mais adequada para a determinação desses potenciais ${ }^{20}$.

A interação entre as partículas da superfície é descrita pela Equação 2, para um conjunto de 16 osciladores harmônicos, como está ilustrado na Figura 3b.

$V_{S U P}=\sum_{i=5}^{20} r_{i} k_{i}^{2}$

Na Equação 2, $r_{\mathrm{i}}$ é a distensão de cada oscilador de sua posição de equilíbrio, calculada a partir das coordenadas de posição $q_{x, y z}$ das partículas ou dos limites da superfície. A constante elástica $k_{i}$ é calculada diretamente a partir das freqüências $v_{1}$ e $v_{2}$ da equação do oscilador harmônico assumindo, na realidade, apenas dois valores $k_{1}$ e $k_{2}$. O potencial total, então, é a soma dos potenciais $V_{S U P} \mathrm{e} V_{I N T} \mathrm{e}$ pode ser expresso pela Equação 3, em função das distâncias entre as partículas, como

$V_{\text {TOTAL }}=V\left(r_{1 \ldots \ldots \ldots} r_{20}\right)$

onde $i=1 \ldots 4$, corresponde à distância átomo-superfície e $i=5 \ldots 20$, aos 16 osciladores da superfície. De forma equivalente, a Equação 3, pode ser expressa em função das coordenadas de posição $q_{i}$, de acordo com a Equação 4:

$V_{\text {TOTAL }}=V\left(q_{1 \ldots \ldots \ldots . .} q_{15}\right)$

\section{O cálculo da dinâmica}

A dinâmica do espalhamento é resolvida através do método da Trajetória Clássica, que calcula as coordenadas de posição $q_{i}(t)$ e de momento $p_{i}(t)$ para cada partícula. $\mathrm{O}$ intervalo de tempo no qual as coordenadas de posição do átomo e das partículas da superfície são registradas, chamado passo de integração, é determinado como um parâmetro externo pelo algoritmo de resolução das equações. Nesse método, as equações do movimento são aquelas derivadas do hamiltoniano $H$ de 15 coordenadas, $q_{i}$ e seus momentos associados, $p_{i}$ como mostra a Equação 5:

$H\left(q_{i}, p_{i}\right)=\frac{1}{2} \sum_{i=1}^{12} \frac{p_{i}^{2}}{m_{i}}+\frac{p_{A}^{2}}{2 m_{A}}+V\left(q_{1}, q_{2}, \ldots \ldots, q_{15}\right)$

Os dois primeiros termos referem-se às energias cinéticas da superfície e do átomo, respectivamente, e o último termo ao potencial total do sistema átomo-superfície em função das coordenadas de posição, como mostra a Equação 4.

Dadas as condições iniciais, onde posição e momento das partículas são selecionados aleatoriamente, a descrição dinâmica do sistema, ou seja, a determinação da posição $q_{i}(t)$ e da nova posição $q_{i}(t$ $+\Delta t$ ) é obtida calculando-se a velocidade, através da Equação 6, e da aceleração e, portanto, da força, que atua sobre a partícula através das Equações 7 e 8.

$\frac{d q_{i}}{d t}=\frac{\partial H}{\partial p_{i}}$ 
$\frac{d p_{i}}{d t}=-\frac{\partial H}{\partial q i}=\frac{\partial V}{\partial q_{i}}$

$\frac{\partial V}{\partial q_{i}}=\sum_{k=1}^{20} \frac{\partial V}{\partial R_{k}} \frac{\partial R_{k}}{\partial q_{i}}$

A resolução de todas as equações de movimento é bastante longa e não será apresentada aqui. Uma descrição mais detalhada deste método e demonstração da derivação das Equações clássicas de movimento pode ser encontrada em Karplus e colaboradores ${ }^{21}$.

As condições finais são determinadas quando a trajetória atinge um tempo limite ou quando o átomo se afasta da superfície o suficiente para não sofrer interação considerável.

Os átomos atingem a superfície com um ângulo de incidência $\theta_{i}$ em relação à normal e são espalhados em um ângulo $\theta_{\mathrm{f}}$, como mostra a Figura 5. O ângulo $\phi$ mede o afastamento da trajetória do átomo incidente para fora do plano da colisão. Na prática, o espalhamento é considerado fora do plano quando $10^{\circ}<\phi<170^{\circ}$. As condições iniciais são selecionadas aleatoriamente, situando o átomo longe o bastante da superfície, de tal modo que a energia potencial de interação gás-superfície seja menor que $10^{-8} \mathrm{eV}$. Observada esta condição e especificada uma direção inicial $\theta_{\mathrm{i}}$, o átomo pode atingir a superfície em qualquer ponto desta. As coordenadas de posição e seus momentos conjugados iniciais, de cada grupo $\mathrm{CH}_{3}$ da superfície, são também gerados aleatoriamente. A energia total é a soma das energias dos modos vibracionais da superfície obtida analiticamente ${ }^{22}$ para o conjunto de 16 osciladores da Figura 1, como mostra a Equação 9.

$E=6\left(n_{x}+1 / 2\right) \hbar v_{x}+6\left(n_{y}+1 / 2\right) \hbar v_{y}+4\left(n_{z}+1 / 2\right) \hbar v_{z}$

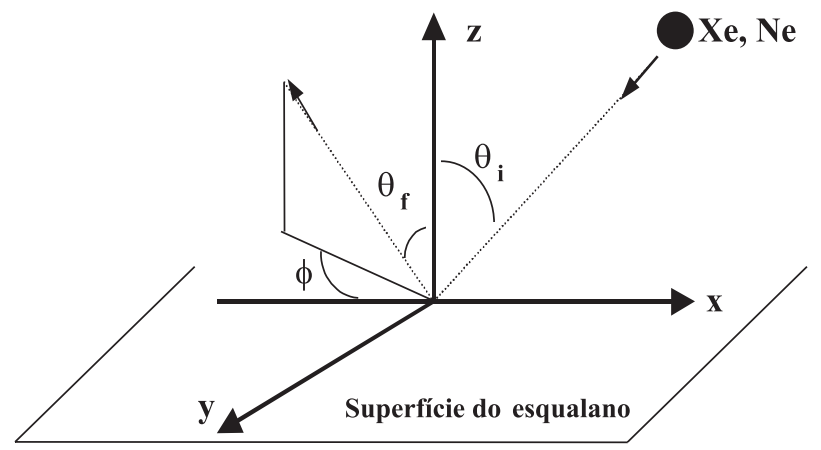

Figura 5. Modelo esquemático para o espalhamento de átomos de Xe e Ne sobre a superfície do esqualano. O ângulo de incidência é $\theta_{i} e$ o de espalhamento é $\theta_{f}$. $\phi$ é o ângulo de espalhamento fora do plano
Os valores da energia cinética e do ângulo de incidência iniciais dos átomos de Ne e Xe aqui empregados são idênticos aos citados na literatura para experimentos de espalhamento ${ }^{8,10} ; \mathrm{E}_{\mathrm{Xe}}=180 \mathrm{~kJ} / \mathrm{mol} \mathrm{e}$ $\theta_{\mathrm{i}}=45^{\circ}, \mathrm{E}_{\mathrm{Ne}}=25 \mathrm{~kJ} / \mathrm{mol}$ e $\theta_{\mathrm{i}}=45^{\circ}$.

Um total de $\mathrm{N}=1000$ trajetórias foram calculadas, com um erro estatístico $\lambda$, máximo, obtido da Equação 10,

$\lambda=\left[\mathrm{p}_{\mathrm{a}}\left(1-\mathrm{p}_{\mathrm{a}}\right) / \mathrm{N}\right]^{1 / 2}$

de $1,5 \%$ para uma probabilidade de adsorção $\mathrm{p}_{\mathrm{a}}=\mathrm{N}_{\mathrm{a}} / \mathrm{N}=35 \%$, onde $\mathrm{N}_{\mathrm{a}}$ representa o número de trajetórias nas quais o átomo é adsorvido pela superfície ${ }^{23}$. O valor de $\mathrm{N}_{\mathrm{a}}$ é obtido calculando-se o tempo médio $t$ para todas as trajetórias e classificando como adsorção, a trajetória cujo tempo de duração é maior que $t$ e como espalhamento inelástico aquelas cujo tempo de duração é menor que $t$. O tempo de trajetória é o tempo medido a partir do instante inicial até que a distância do átomo à superfície seja igual ou maior à distância inicial. $\mathrm{O}$ valor de $\lambda$ assegura a acurácia estatística dos resultados obtidos a partir desse critério de classificação da colisão em espalhamento inelástico ou adsorção.

\section{RESULTADOS E DISCUSSÃO}

Resultados experimentais do espalhamento de átomos de $\mathrm{Ne}$ pela superfície do esqualano ${ }^{10}$ mostram que, em média, $36 \%$ da energia cinética de $25 \mathrm{~kJ} / \mathrm{mol}$ do átomo incidente é absorvida pelo líquido na colisão. O resultado obtido com o modelo aqui empregado é de $29 \%$, um valor um pouco abaixo do resultado experimental. Verifica-se ainda, experimentalmente, através da distribuição da energia translacional final dos átomos que, em $73 \%$ das colisões ocorre o espalhamento inelástico e em $27 \%$, a adsorção dos átomos na superfície. Neste trabalho, foi determinado que $71 \%$ do espalhamento é inelástico e $29 \%$ é adsorção, valores bastante satisfatórios levandose em conta a simplicidade do modelo da superfície. Esses resultados indicam que, para a energia cinética inicial do átomo de $25 \mathrm{~kJ} / \mathrm{mol}$, a malha de osciladores pode absorver uma parte significativa da energia na colisão e ativar alguns de seus modos vibracionais, cujas frequiências fundamentais sejam próximas daquelas dos modos vibracionais de menor energia do polímero correspondente. Esses resultados são também evidenciados nos histogramas de distribuição da energia final dos átomos adsorvidos ou espalhados inelasticamente, mostrados nas Figuras 6a e b.

Na Figura 6a, que ilustra a adsorção, pode-se observar que a energia final média do átomo fica entre $10 \%(2,5 \mathrm{~kJ} / \mathrm{mol})$ e $70 \%$ $(17,5 \mathrm{~kJ} / \mathrm{mol})$ de seu valor inicial, enquanto que no caso do espalhamento inelástico, mostrado na Figura 6b, este valor situa-se entre 70 e $100 \%$. Esses resultados estão de acordo com o observado
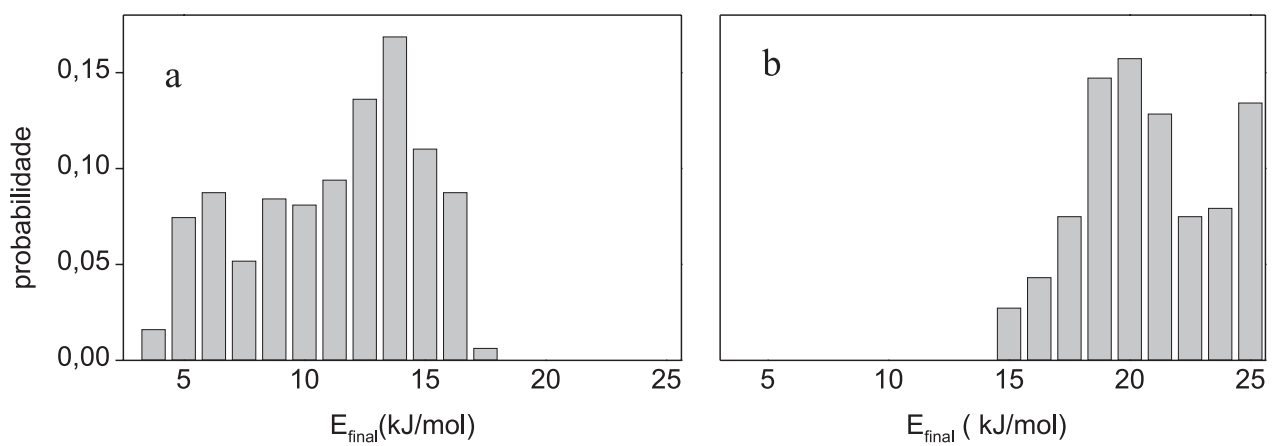

Figura 6. Distribuição da velocidade final dos átomos de Ne na colisão com a superfície do esqualano na captura e dessorção, 6a, e no espalhamento inelástico, 6b. A energia inicial é de $25 \mathrm{~kJ} / \mathrm{mol}$ 
experimentalmente ${ }^{10} \mathrm{e}$ confirmam que o critério de distinção entre os eventos espalhamento inelástico e captura e dessorção reproduz satisfatoriamente as características dinâmicas da colisão.

A influência da temperatura da superfície na dinâmica do espalhamento gás-líquido tem sido objeto de investigação em diversos trabalhos experimentais ${ }^{11-13}$. Em particular, Nathanson e colaboradore ${ }^{11}$ mostraram que o aumento da temperatura da superfície de líquidos orgânicos favorece a adsorção temporária de átomos de Xe, aumentando ligeiramente a captura e dessorção do gás na superfície líquida. Segundo os autores, essa tendência de favorecimento à adsorção está relacionada à maior amplitude de deslocamento no movimento dos grupos da superfície, o que facilita o aprisionamento das espécies que colidem. Essa é uma explanação bastante genérica pois, na realidade, a dinâmica da adsorção de um gás na superfície líquida é um processo complexo e não há um cenário único que possa descrevê-lo. Esse resultado é qualitativamente confirmado neste trabalho. $\mathrm{O}$ aumento da temperatura da superfície real do esqualano é analisado através do aumento da sua energia inicial, determinada para valores de $n_{x}, n_{y}$ e $n_{z}$, na Equação 9, de 0 a 4 . Os valores percentuais dos dois processos de colisão inelástica e adsorção estão mostrados na Tabela 1, para o átomo de $\mathrm{Ne}$ com energia inicial de $25 \mathrm{~kJ} / \mathrm{mol}$. Como se pode observar, valores crescentes de $n=n_{x}, n_{y} \mathrm{e}$ $n_{z}$ provocam o favorecimento da adsorção e a diminuição na probabilidade de colisão inelástica. Este efeito não é muito significativo do ponto de vista quantitativo, o que também foi observado experimentalmente $^{11}$, mas confirma que este modelo de representação da superfície líquida permite reproduzir as características dinâmicas importantes do processo de espalhamento gás-líquido.

Tabela 1. Percentual de átomos espalhados inelasticamente e adsorvidos. Os diferentes valores de $n$ estão relacionados às temperaturas crescentes da superfície

\begin{tabular}{ccc}
\hline $\mathrm{n}$ & C. inelást. $(\%)$ & Adsorção(\%) \\
\hline 0 & 69,2 & 30,8 \\
1 & 66,1 & 33,9 \\
2 & 66,2 & 33,7 \\
3 & 66,3 & 33,8 \\
4 & 64,3 & 35,7 \\
\hline
\end{tabular}

Outro efeito da temperatura da superfície no espalhamento de átomos no caso de líquidos metálicos, observado por Ronk e colaboradores $^{11}$, é uma pequena diminuição no espalhamento especular onde $\phi \cong 0^{\circ}$ e $\theta_{\mathrm{i}} \cong \theta_{\mathrm{f}}$ na Figura 5 .

Resultados experimentais do padrão de espalhamento angular permitem caracterizar a irregularidade da superfície e mostram dependência com o ângulo de incidência $\theta_{\mathrm{i}}$ do átomo. Lipkin e colabo- radores $^{8}$ observaram que os átomos de Xe são massivamente espalhados pela superfície do esqualano para fora do plano da colisão, ou seja o ângulo $\phi$ que mede o espalhamento para fora do plano situa-se no intervalo $10^{\circ}<\phi<170^{\circ}$, como indicado na Figura 5. Para átomos que atingem a superfície em direções quase paralelas à esta, a tendência de realizar múltiplas colisões na superfície e ser espalhado fora do plano é pequena. Neste trabalho, a distribuição de átomos espalhados fora do plano para um ângulo de incidência $\theta_{i}=70^{\circ}$ é desprezível. Para $\theta_{\mathrm{i}}=45^{\circ}$ a distribuição de átomos espalhados pela superfície é mais homogênea, com uma certa predominância para $\phi=45^{\circ}$, similar à distribuição obtida com outro modelo para superfície do esqualano, como mostra a Figura 7.

As Figuras 8a e b mostram a variação temporal da distância do átomo à superfície, ou $\mathrm{z}(\mathrm{t})$, de acordo com a Figura 5, para uma tripla colisão verificada em uma trajetória particular. A duração desse evento é de, aproximadamente, 2 picossegundos, resultado semelhante ao obtido na simulação do espalhamento de átomos de $\mathrm{Ar}$ pela superfície do In líquido. Experimentalmente, observa-se ainda que o número total de colisões múltiplas pode atingir até 10 , o que não pôde ser verificado neste trabalho, devido à limitação das dimensões físicas do modelo de superfície utilizado ${ }^{8}$.

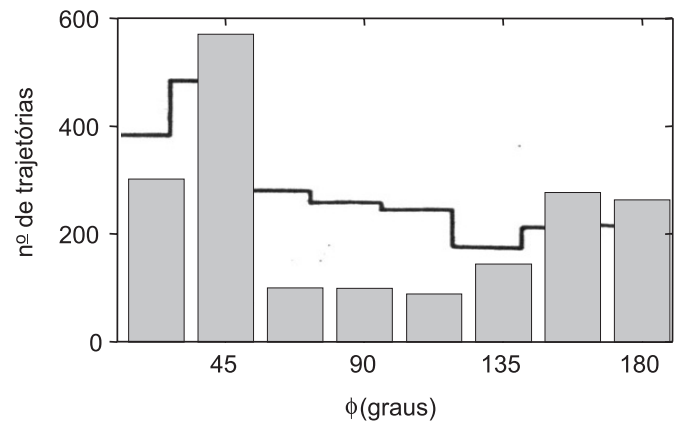

Figura 7. Distribuição do ângulo de espalhamento fora do plano ( $\phi)$, de acordo com a Figura 5, para a colisão Xelesqualano calculado com o modelo proposto neste trabalho (gráfico em barra) e de acordo com o modelo proposto na ref. 8 (linha contínua). A energia inicial do átomo é de $180 \mathrm{~kJ} /$ mol e o ângulo de incidência, $45^{\circ}$

\section{CONCLUSÃO}

O modelo desenvolvido para o estudo da dinâmica da colisão de átomos com superfície de um líquido polimérico reproduz com boa concordância os resultados experimentais conhecidos.

Os histogramas de distribuição de energia para a colisão Ne-esqualano mostram que quando o átomo é adsorvido sua energia final está entre 10 e $70 \%$ de sua energia inicial, enquanto que no
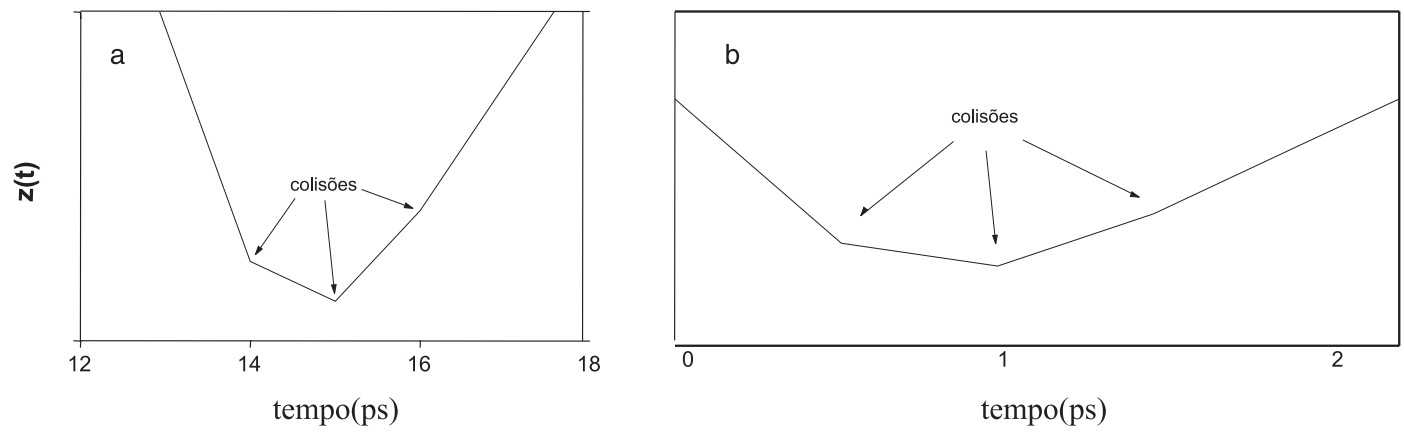

Figura 8. Evolução temporal da distância átomo-superfície para uma energia inicial de $25 \mathrm{~kJ} / \mathrm{mol}$ para a) o sistema Ne-esqualano calculada neste modelo e b) Ar-In líquido com o modelo proposto na ref. 9 
espalhamento inelástico esta energia varia de 70 a $100 \%$ da sua energia inicial, o que concorda com os resultados observados experimentalmente. $\mathrm{O}$ aumento da fração dos átomos incidentes que são adsorvidos na colisão com o aumento da temperatura da superfície líquida, observado experimentalmente, é também verificado qualitativamente através do aumento da probabilidade de adsorção com o aumento da energia inicial da superfície modelo. Esse resultado sugere que a turbulência das moléculas na superfície líquida, que ocorre com o aumento da temperatura, descrita aqui pela maior oscilação dos grupos proeminentes pode, pelo menos em princípio, ser obtida com esta representação simplificada da superfície.

A distribuição angular dos átomos de Xe espalhados fora do plano é similar ao resultado experimental ${ }^{8}$, o que sugere que a colisão é um evento localizado, do qual participam poucos átomos ou moléculas da superfície.

Observando-se trajetórias típicas nas quais ocorrem múltiplas colisões com a superfície, é possível determinar o tempo total das colisões múltiplas, que é da mesma ordem daquele observado no espalhamento entre átomos e superfícies metálicas líquidas.

Esses resultados sugerem que o modelo de superfície líquida, descrita como uma malha de osciladores acoplados com freqüências de vibração correspondentes às freqüências dos modos vibracionais de mais baixa energia do líquido, pode descrever corretamente a dinâmica do espalhamento gás-líquido. A descrição satisfatória das características dinâmicas da colisão entre átomos de Ne e Ar e a superfície líquida de esqualano indicam que essa é uma direção promissora na pesquisa deste tema. Os passos seguintes devem considerar uma melhor caracterização do potencial de interação gás-superfície, incluindo os termos de interação de três corpos e a descrição mais realística da própria superfície, com a inclusão de maior número de osciladores.

\section{AGRADECIMENTOS}

Ao CNPq e à FAPEMIG pelo apoio financeiro.

\section{REFERÊNCIAS}

1. Waclawik, E. R; Cynthia-Goh, M.; Donaldson, D. J.; J. Chem. Phys. 1999, 110,8798 .
2. Karpovich, D. S.; Ray, D.; J. Phys. Chem. B 1998, 102, 650.

3. Jayne, J. T.; Worsnop, D. R.; Kolb, C. E.; Swartz, E.; Davidovits, P.; J. Phys. Chem. 1996, 100, 8015.

4. http://www.chem.wisc.edu/ nathanson/, acessada em Setembro 2000.

5. Heal, M. R.; Pilling, M. J.; Titcombe, P. E.; Whitaker, B. J.; Geophys. Res. Lett. 1995, 22, 3043.

6. Gliss, P. S.; Slater, P. G.; Nature 1974, 25, 181.

7. Scharlin, P.; Battino, R.; Silla, E.; Tunon, I.; Pascual-Ahuir, J. P.; Pure Appl. Chem. 1998, 70, 1895.

8. Lipkin, N.; Gerber, R. B.; Moiseiev, N.; Nathanson, G. M.; J. Chem. Phys. 1994, 100, 8048.

9. Tribe, L.; Manning, M.; Morgan, J. A.; Stephens, M. D.; Ronk, W. R.; Treptow, E.; Nathanson, G. M.; Skinner, J. L.; J. Phys. Chem. B 1998, 102, 206

10. Saecker, M. E.; Govone, S. T.; Kowalsky, D. V.; King, M. F.; Nathanson, G. M.; Science 1991, 252, 1421.

11. Ronk, W. R.; Kowalski, D. V.; Manning, M.; Nathanson, G. M.; J. Chem. Phys. 1996, 104, 4842; Nathanson, G. M.; Davidovits, P.; Worsnop, A. R.; Kolb, C. E.; J. Phys. Chem. 1996, 100, 13007.

12. Keynon, A. J.; McCaffery, A. J.; Quintella, C. M.; J. Chem. Soc. Faraday Trans. 1993, 89, 3877.

13. Quintella, C. M.; McCaffery, A. J.; Zidan, M. D.; Chem. Phys. Lett. 1993, $214,563$.

14. Leal, A. S.; dos Santos, C. G.; Quintella, C. M.; Schor, H. H. R.; J. Braz. Chem. Soc. 1999, 10, 359.

15. Leal, A. S.; Tese de Doutorado, Universidade Federal de Minas Gerais, Brasil, 2001.

16. Albert, N. L.; Keiser, W. E.; Szymansky, H. A.; Theory Practice of IR Spectroscopy, ed. Plenum Press: New York, 1970.

17. Mclaughin, D. R.; Thompson, D. L.; J. Chem. Phys. 1973, 50, 4393; Greeley, J. N.; Martin, J. S.; Morris, J. R.; Jacobs, D. C.; J. Chem. Phys. 1995, 102, 4996; Vach, H.; Hager, J.; Walter, H.; Chem. Phys. Lett. 1987, $133,279$.

18. Liuti, G.; Pirani, F.; Buck, U.; Schmidt, B.; Chem. Phys. 1998, 126, 1.

19. Chase, D.; Manning, M.; Morgan, J. A.; Nathanson, G. M.; Gerber, R. B.; J. Chem. Phys. 2000, 113, 9279.

20. Tantardini, G. F.; Simoneta, M.; Surf. Sci. 1978, 112, 337; Ramasamy, S.; Pradeep, T.; J. Chem. Phys. 1995, 103, 485; Tantardini, G. F.; Simoneta, M.; Chem. Phys. Lett. 1982, 87, 420; Sander, L. M.; Ghaisas, S. V.; Surf. Sci. 1997, 391, 125; Weare, J. H.; Potential Energy Surfaces and Dynamics Calculations, ed. Plenum Press: New York, 1976; Porter, R. N.; Raff, L. M.; Dynamics of Molecular Collisions, Part B, ed. Plenum Press: New York, 1976.

21. Karplus, M.; Porter, R. N.; Symon, K. R.; Sharma, R. D.; J. Chem. Phys. 1963, 43, 3259.

22. Symon, K. R.; Mechanics, ed. Addison Wesley Co.: Tokyo, 1963.

23. Pazzi, V. I.; Tantardini, G. F.; Surf. Sci. 1997, 377, 572. 